\title{
Chinese astronomers look to Antarctic
}

A Chinese expedition returned last week from a 14-day crawl across the East Antarctic ice sheet in cargo containers, pulled by tractors, that doubled as living quarters. The trip, sponsored by the Polar Research Institute of China, completes only the second traverse to Dome A - the highest point on the eastern ice cap and the place where China intends to start building a research base next year.

The team also set up a suite of research instruments to study the atmosphere and sky above Dome A, most notably a remotely operated observatory called PLATO, which will assess how good the skies are for astronomical 'seeing. PLATO includes four 14.5-centimetre telescopes, built in China, that will take advantage of more than three straight months of darkness during the Antarctic winter. "We think Dome A is the best site on Earth for astronomy," says Xiangqun Cui of the Nanjing Institute of Astronomical Optics and Technology.

The hope is that the desolate plateau, which sits 4,100 metres above sea level, will boast conditions unrivalled elsewhere on the planet - even at the French/Italian base at Antarctica's Dome C, 1,200 kilometres away, which set up its own automated test observatory in 2003 and has since ramped up to larger projects.

Proponents of Antarctic astronomy have looked to Domes A and C as alternative sites to the South Pole, above which 300 metres of turbulent air cause observations of stars to jitter and blur ${ }^{1}$. Dome C, by contrast, has only 30 metres of turbulent air above it, and less atmospheric interference than astronomical observing sites in Hawaii and Chile 2 .

Although it lacks infrastructure, Dome A, which sits some 900 metres higher than Dome $\mathrm{C}$, could be an even more promising site. "Each

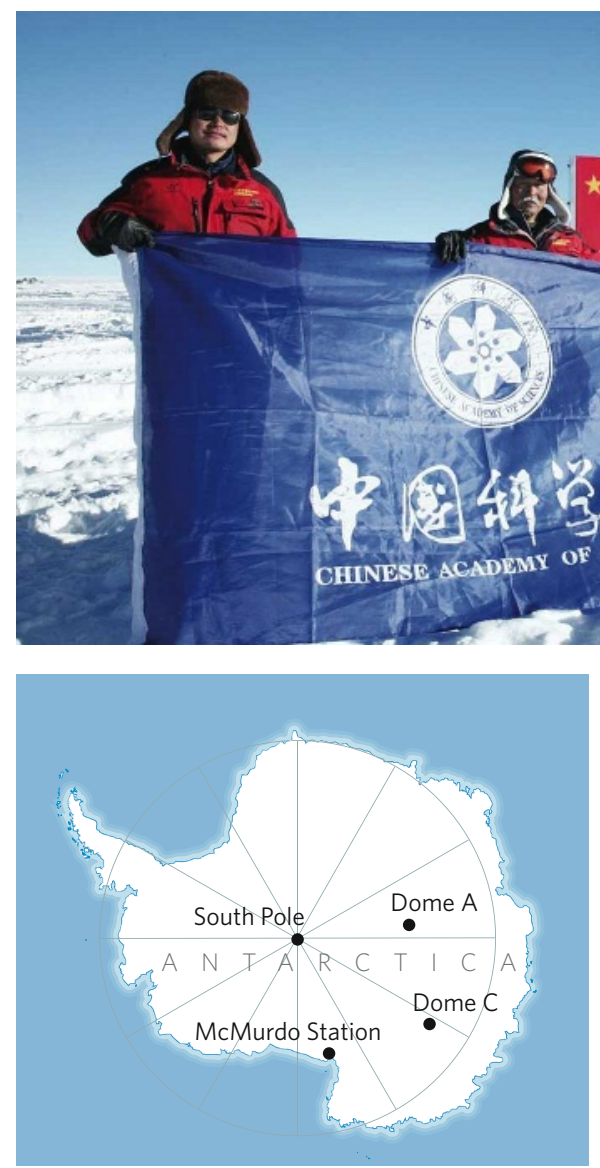

additional 100 metres is very important because it knocks out a huge chunk of the atmosphere," says Michael Ashley of the University of New South Wales in Sydney, which built PLATO. Models suggest that Dome A may have a turbulent layer as thin as 5 metres, he says, and thus even better seeing.

\section{Stars on ice}

Most astronomical projects in Antarctica are based at the South Pole. They include:

The US 10-metre South Pole Telescope, which saw first light in February 2007, scans the sky in the millimetre and sub-millimetre range, focusing primarily on the cosmic microwave background left over from the Big Bang.

IceCube, the underground US neutrino observatory, reached its halfway point in construction this Antarctic summer; completion is set for 2011.

At the McMurdo Station in the Ross Sea, the United States launches payloads aboard scientific balloons reaching up to 42 kilometres. This year's missions include US and Japanese instruments examining cosmic rays and antimatter.

At the more recently established Dome C, projects include:

Italy's Small IRAIT, a 25-centimetre prototype for an 80-centimetre microwave telescope, was installed last year to study objects such as brown dwarfs in the Milky Way.

France's ASTEP, a

40-centimetre telescope dedicated to photometry and exoplanet searches, is scheduled to be mounted on Dome C in January 2009. R.C.
Cold comfort: China has set up a remotely operated observatory on Dome A, the summit of East Antarctica's ice cap.

Such conditions make Dome A attractive to Chinese astronomers, who have begun work on a suite of three 0.5 -metre telescopes that they hope to deploy at the site in 2009. They are also eyeing the location for a potential US\$40-million, 4-metre infrared and optical telescope. A proposal on that may be submitted this summer to the Chinese Academy of Sciences.

The true potential of Dome A may lie in observations outside optical wavelengths. The efficiency of infrared astronomy is particularly sensitive to temperature, and winter nights that drop as low as $-90^{\circ} \mathrm{C}$ will eliminate much of the noise from the atmosphere and the telescope itself, researchers say.

The potentially unparalleled dryness of the air may also allow astronomers to access parts of the electromagnetic spectrum that are obscured by water vapour elsewhere, including Dome C. "We're hoping to observe at very high radio frequencies that you would only otherwise be able to do in a plane or in space," says Craig Kulesa of the University of Arizona, who has an instrument on PLATO that will assess the transparency of the atmosphere. If conditions are as dry as expected, Kulesa hopes to deploy a telescope to Dome A that will map the Milky Way in the far infrared, to learn more about star formation.

But future projects will depend on PLATO, which researchers hope will yield meaningful data before next year's trip to refuel the station. "We already know Dome A will be the best spot on Earth," say Kulesa. "But the question is, how good is that?"

\section{Rachel Courtland}

1. Travouillon, T. et al. Astron. Astrophys. 409, 1169-1173 (2003). 2. Lawrence, J. S. et al. Nature 431, 278-281 (2004). 